Conclusion In order to identify more rapidly a wide variety of mycobacteria, the PCR-restriction fragment length polymorphism analysis of $h s p 65$ procedure was applied. The PRA test among NTM isolates indicated that the most frequent mycobacterial strains were $M$. kansasii, $M$. gordonae III, $M$. marinum, $M$. chelonae, $M$. scrofluaceum and $M$. gastri. Our results showed that this method in comparison with classical methods is rapid and accurate enough for the identification of mycobacterial species from LJ culture isolates.

\section{MOLECULAR CHARACTERISTICS OF METHICILLIN RESISTANT STAPHYLOCOCCUS AUREUS ISOLATED FROM CHINESE CHILDREN}

doi:10.1136/archdischild-2012-302724.0273

X Shen, Y Liu. Beijing Children's Hospital, Capital Medical University, Beijing, China

Objective The present study aim to investigate the molecular characteristics of methicillin-resistant Staphylococcus aureus (MRSA) isolates from Chinese children in seven city.

Method A total of 134 MRSA isolats were collected from eight hospitals. Multilocus sequence (MLST), staphylococcal chromosomal cassette mec (SCCmec) and spa typing were analyzed. The PantonValentine leukocidin (pvl) gene was also detected.

Result Overall, 16 sequence types (STs) were obtained, and CC59 $(51.7 \%)$ was found to be the most prevalent, which including ST59 and ST338, followed by ST239 (16.4\%). SCCmec type II, III, IV and $\mathrm{V}$ were identified in this study. SCCmec type IV was the most predominant type at $50.0 \%$, followed by SCCmec type $\mathrm{V}$ at $23.9 \%$ and III at $23.9 \%$. SCCmec subtypes IVa, IVc, and IVg were found among the SCCmec type IV strains, IVa was the main subtype at $77.6 \%$. Twenty-six spa types were also identified, the predominant type was $t 437(47.8 \%)$. The prevalence of pvl genes and the SCCmec type of the strain were relevant, the pvl gene positive rate was higher in SCCmecIV and V-type strains than in SCCmecII and IIItype strains $(58.6 \%$ vs. $14.3 \%, \mathrm{P}<0.05)$; there was a significant difference between them. In the strains isolates from pneumonia and SSTIs, ST59-MRSA-Iva (t437) was the predominnant clone.

Conclusion The result indicates that MRSA isolates in Chinese children are largely associated with the ST59-MRSA-IV (t437) and ST239-MRSA-III (t037) clone.

\section{COLONIZATION OF METHICILLIN-RESISTANT STAPHYLOCOCCUS AUREUS WITH HIGH-LEVEL RESISTANCE TO MUPIROCIN IN KOREAN CHILDREN}

doi:10.1136/archdischild-2012-302724.0274

JH Lee, SJ Kim, J Lee, HS Lee, SY Kim, IK Sung. Department of Pediatrics, The catholic University of Korea, Seoul, Republic of Korea

Increased mupirocin use has been considered as a major cause to develop mupirocin resistance among MRSA isolates. High-level mupirocin resistance (HLMR) is associated with decolonization failure, but fortunately most MRSA have showed low-level mupirocin resistance (LLMR). Recently, we became aware of markedly high prevalence of clinical isolates with HLMR among Korean children. We investigated the proportion of HLMR isolates and mupirocin use between children (less than 18 years) and adult patients.

A total of 1134 MRSA (213 children and 921 adults) isolates were identified from two university hospitals in 2010-2011. Antimicrobial susceptibility testing was firstly performed by using the Vitek 2 instrument (bioMerieux, Marcy I'Etoile, France). The mupA genes were detected by polymerase chain reactions.

Overall, 38\% of isolates collected from children showed HLMR, whereas $3.4 \%$ showed HLMR in adults. Children revealed only one LLMR isolate $(0.4 \%)$, and adult patients had 80 LLMR isolates (8.6\%). This tendency was also observed, when the patients were divided into two groups under intensive care units or outpatient settings. A total of 4,009 mupirocin prescriptions were dispensed at our institutions during 2006. Afterward, there were 4,760, 5,250, 6,416, and 8,038 prescriptions from 2007 to 2010, respectively. But, prescription rates of mupirocin did not significantly differ between children and adults. In children with MRSA isolates, the presence of previous admission, prolonged hospitalization, and mupirocin use did not contribute to mupirocin resistance. In Korean children, the rate of HLMR in the MRSA isolates was very high and it was not associated with increased mupirocin use.

\section{EPIDEMIOLOGY OF POLIOMYELITIS AND MANAGEMENT OF ACUTE ASCENDING FLACCID PARALYSIS (AAFP) IN PAEDIATRIC INTENSIVE CARE UNIT (PICU) IN ALGERIA}

doi:10.1136/archdischild-2012-302724.0275

MA Negadi, H Bouguetof, K El Halimi, D Boumendil, ZC Mentouri. Pediatric Intensive Care Unit, Faculty of Medicine, Oran University, Oran, Algeria

Background and Aims As part of the implementation of National plan for the eradication of poliomyelitis, disease surveillance and management of cases of AAFP are key strategies of this program.

The aim is participation in the national program for poliomyelitis, eradication and to determine the efficacy of intravenous immunoglobulin (IG) for treatment of AAFP.

Methods From September 1994 to February 2012, 176 cases of AAFP were identified and supported in our PICU. Since January 2008, 25 children were treated by IG. The parameter studied were: age, gender, autonomic disorders, albuminocytologic dissociation of CSF after lumbar puncture, artificial ventilation, average time of stay, and mortality.

Results In this series, no case of poliomyelitis has been diagnosed by the Pasteur Institute of Algeria. Since 2008, use of IG seems to improve the mortality. $8 \%$ in the overall series $(n=176)$ vs $4 \%$ in the group treated with immunoglobulins $(n=25)$. All deaths are related to the existence of severe neurovegetative disorders. The need in ventilation and the duration of stay does not appear to be influenced by the use of the "IG"

Conclusion The eradication of poliomyelitis is a national goal to declare the Algeria zone free of polio by the WHO.

\section{INFANT MORTALITY FROM INFECTION OVER 2 DECADES: LESS GBS AND MENINGOCOCCUS, BUT DOUBLING OF DEATHS IN VERY PRETERM INFANTS}

doi:10.1136/archdischild-2012-302724.0276

'EJ Williams, 1,2ND Embleton, ${ }^{3} \mathrm{M}$ Bythell, 1,2JE Berrington. 'Neonatal Medicine, Newcastle Upon Tyne Hospitals NHS Foundation Trust; ${ }^{2}$ Institute of Health and Society; ${ }^{3}$ Regional Maternity Survey Office, Newcastle University, Newcastle Upon Tyne, UK

Background Infection is an important cause of neonatal/infant mortality. Antenatal care, neonatal intensive care and immunisation practices affect infectious mortality, but no good data show how these deaths have changed over time. Understanding this will help direct future medical priorities.

Objective To evaluate changes in neonatal/infant mortality from infection over 2 decades (1988-2008) in a geographical population. Design and Methods We used a population database (Perinatal Mortality Survey, Northern region UK) and reviewed infant deaths coded as infection. Proportional contribution to deaths, pathogens identified and risk factors were analysed. To demonstrate changes over time, three 7-year epochs were created.

Results 625 deaths from infection were identified. Absolute numbers of deaths fell with time but the proportion from infection increased. Significantly preterm infants were increasingly represented in successive epochs. 